\title{
Earthquake Time Series Analysis by Cantor Set Model in Taiwan
}

\author{
Yen-Chieh Chen ${ }^{1}$, Quo-Cheng Sung ${ }^{2,{ }^{*}}$, Ting-To $\mathrm{Yu}^{1}$, and Robert J. Sun ${ }^{1}$
}

(Manuscript received 26 July 2002, in final form 10 February 2003)

\begin{abstract}
The Cantor set fractal distribution and probability model are combined to analyze earthquake time series of two seismogenic zones of Taiwan from 1900 to 1999. One-dimensional box counting method is adopted to measure the occurrence probability of earthquakes equal to or larger than a certain magnitude threshold with different time scales. The results show that the fractal dimension has a negative correlation with the magnitude threshold, and the ideal critical time scale has an exponential correlation with the magnitude threshold. The ideal critical time scale can be deemed as the "lower limit" of recurrence period of earthquake clustering that can be referenced in designing earthquake resistant construction. Due to frequent and continuous clustering of earthquakes occurred in the Chianan zone, we suggest that the fault activities in the Chianan zone are more active than that in the Center zone of Taiwan.
\end{abstract}

(Key words: Fractal, Earthquakes of Taiwan, Clustering, Cantor set)

\section{INTRODUCTION}

It is important to understand the temporal fractal behavior of earthquake occurrence when analyzing earthquake time series (Smalley et al. 1987; Ogata 1988; Turcotte 1992; Papadopoulos and Dedousis 1992; Chen et al. 1998; Tsai 1999). Gutenberg and Richter (1944) first noticed that the relation between cumulative earthquake frequency $(N)$ and magnitude $(M)$ is $\log N=\mathbf{a}$ - b $\times$ M. Studies show that the $\mathbf{b}$ value is usually between $0.8-1.2$, whereas the a value is a region-dependent measurement (Evernden 1970; Wang 1991). After Mandelbrot (1967) introduced the concept of fractal geometry, Aki (1981) summarized a relation between the b value and the fractal dimension (D) to be $\mathbf{b}=\mathrm{D} / 2$, and this result indicated the self-similar fractal characteristic of earthquake time series. Kagan and Jackson (1991) found that long-term earthquake clustering characterizes the occurrence of earthquakes and that the fractal dimension of the earthquake time series is in the order of $0.8 \sim 0.9$. Wang $(1995,1996)$ claimed the

\footnotetext{
${ }^{1}$ Department of Earth Sciences, National Cheng Kung University, Tainan, Taiwan

${ }^{2}$ Department of Geography, National Kaohsiung Normal University, Kaohsiung, Taiwan

* Corresponding author address. Prof. Quo-Cheng Sung, Department of Geography, National Kaohsiung Normal University, Kaohsiung, Taiwan, ROC; E-mail: sungqc@ nknucc.nknu.edu.tw
} 
multifractality of earthquake time series after analyzing data from mainland China and Taiwan using a generalized correlation time integral $\left[\mathrm{C}_{\mathrm{q}}(\mathrm{t})\right]$. The Cantor-set type clustering is characteristic to many natural phenomena. Smalley et al. (1987) concluded that earthquake clustering in time is a scale-invariant process, and that the earthquake time distribution is certainly different from Poisson distribution. Mayer (1992) analyzed desert storm sequence and found that storm data is self-similar with different rainfall scales and that the fractality of storm clustering prevails in scale of decades. Olsson et al. (1992) and Lin (1998) confirmed that rainfall time series are scale-invariant in time, and Mazzarella (1998) obtained a similar result when analyzing sea flooding's catalog in Venice. Chen et al. (1998) and Tsai (1999) provided a sieve (or threshold) method for describing fractal-clustering variation of earthquakes. Lin (1998) and Tsai (1999) further developed probabilistic concept to construct the recurrence period of natural events.

Recurrence period is a very important parameter, especially for natural catastrophes. In seismic hazard evaluation, it can be a critical reference value in designing the aseismatic strength of buildings or civil constructions during their designed life. This study applies threshold and probability methods to compute the fractal dimension and the recurrence periods of earthquake clustering in time domain for two seismogenic zones in Taiwan. Further more, a new effective method for measuring the recurrence period will also be given and discussed in details.

\section{THEORY AND METHODS}

\subsection{Similarity Dimension and Box-Counting}

Self-similarity and scale-invariance are the basic concepts of fractal geometry. Suppose a $\mathrm{D}$-dimensional cube in Euclidean space is divided into $\mathrm{N}$ equal small cubes. In such a way, every side of the original cube is divided into $\mathrm{m}$ divisions. As a ratio scale set to be $\mathrm{r}=1 / \mathrm{m}$, then:

$$
\begin{aligned}
& \text { if } D=1, N=m^{1}=(1 / r)^{1} ; \\
& \text { if } D=2, N=m^{2}=(1 / r)^{2} ; \\
& \text { if } D=3, N=m^{3}=(1 / r)^{3} .
\end{aligned}
$$

For a D-dimensional case,

$$
\mathrm{N}=\mathrm{m}^{\mathrm{D}}=(1 / \mathrm{r})^{\mathrm{D}}
$$

We define the similarity dimension (D) as:

$$
\mathrm{D}=\ln (\mathrm{N}) / \ln (1 / \mathrm{r})
$$

The similarity dimension $\mathrm{D}$ can be either an integer or a fraction.

In practical computation, box-counting method is usually used for obtaining $\mathrm{D}$ values (Rasband 1900; Peitgen et al. 1992; Falconer 1993; Isham 1993; Stoyan and Stoyan 1994; Lee 1995; Cheng et al. 1999). Let $\mathbf{A}$ be a set of finite points within a D-dimensional Euclidean 
Space, and let $\mathrm{N}(\varepsilon)$ be the fewest D-dimensional cubes of size $\varepsilon$ in every side, which can encompass all points of set $\mathbf{A}$. The cube size $(\varepsilon)$ here would be similar to the ratio scale (r), and $\mathrm{N}(\mathcal{E})$ increases as $\varepsilon$ approaches to zero. As $\varepsilon$ approaching zero, $\mathrm{N}(\varepsilon)$ would gradually become close to the exact number of points for set $\mathbf{A}$. So the relationship between small $\varepsilon$ and $\mathrm{N}(\varepsilon)$ is :

$$
\mathrm{N}(\varepsilon) \propto(1 / \varepsilon)^{\mathrm{D}_{\mathrm{B}}} \quad \text { or } \quad \mathrm{D}_{\mathrm{B}}=\lim _{\varepsilon \rightarrow 0} \frac{\ln (\mathrm{N}(\varepsilon))}{\ln (1 / \varepsilon)} .
$$

$D_{B}$ is referred as the "Box-counting Dimension" or "Box-Dimension" and is also generated under the self-similarity concept. It is also easily to understand that by Eq. (1b) and Eq. (2), D and $D_{B}$ of an object with self-similarity would be the same while the ratio scale (r) and the cube size $(\mathcal{E})$ are infinitesimal. The box-counting method has been applied to compute fractal dimensions of natural features in two and three dimensions (Lee 1995; Cheng et al. 1999). In this study, the method is applied to one-dimensional analysis.

\subsection{Cantor Set}

The German mathematician G. Cantor introduced this set in 1883. The Cantor set (also called as Cantor dust) is an infinite point set in the unit interval [0,1]. The set is constructed by initially dividing a line of length 1 into three segments. The middle third is then removed, leaving the two side segments. Divide each remaining segment into three and remove the middle third again. Repeat the process infinite times and the limit will form an infinite number of points while the total length of the undeleted line segments approaching zero. This kind of Cantor set is referred as the deterministic Cantor set (Fig.la) due to its deterministic generation process. If in each step we randomly select one of the three segments to be removed, the resulting set is referred as the random Cantor set (Fig.1b). For both the deterministic and random Cantor sets, $D=\ln 2 / \ln 3 \approx 0.6309$ by Eq. (lb) and Eq. (2) while the measuring size approaches infinitesimal. In practice, the generation process of Cantor set would be finite between 0 and 1 because natural point events in a certain time period are always finite. The random Cantor set produces a distribution of clustering which is usually applied to describe many natural point events clustered irregularly in time, such as rainfall (Lin 1998) or earthquakes (Smalley et al. 1987; Tsai 1999).

\subsection{Fractal Distribution Probability of Point Events}

In this section, we relate fractal distribution to probability (Turcotte 1992). This can be done using the sequence of one-dimensional line segments of the Cantor set illustrated in Fig. 1. The objective is to determine the probability $[\mathrm{P}(\mathrm{r})]$ that a step of ratio scale $\mathrm{r}$ will include a line segment. At step zero, the number of line segment is $\mathrm{N}=1$ and the probability that a set of ratio scale $r=1$ will include a line segment is $P(r)=1$; at stepl, we have $N=2, r=1 / 3$ and $P$ $(r)=2 / 3$, and at step2, $N=4, r=1 / 9$ and $P(r)=4 / 9$ (Figs. $1 \mathrm{a}, \mathrm{b}$ ). So the probability $[\mathrm{P}(\mathrm{r})]$ can be generalized to: 
TAO, Vol. 14, No. 1, March 2003

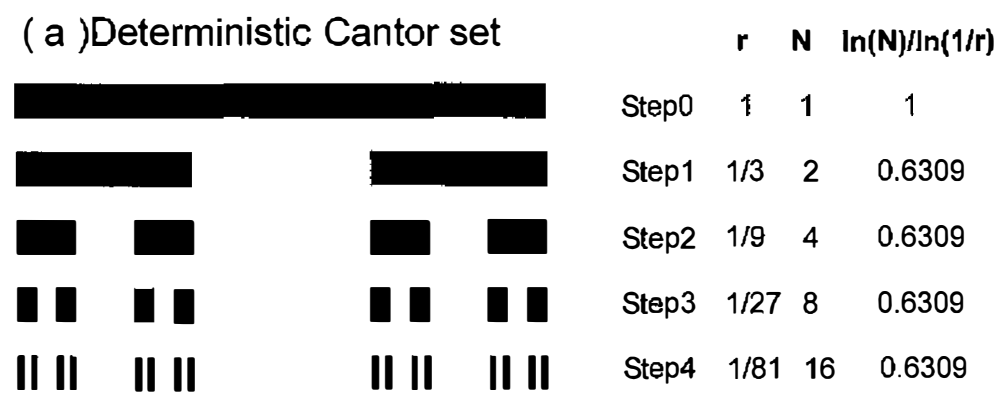

(b) Random Cantor set

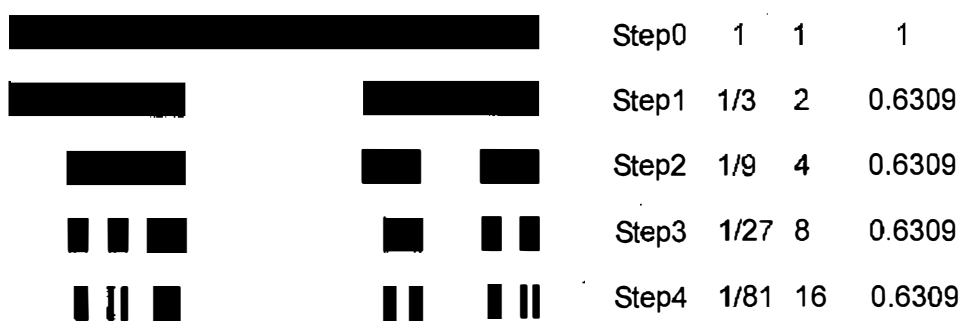

Fig. 1. Three segments Cantor set produced after four repeating processing of "Generator (Step1)" from "Initiator (Step0)". (a) Deterministic Cantor set and (b) Random Cantor set. The length of the undeleted line segments is just for enhancing the point set. The fractal dimensions for both sets are the same $(\approx 0.6309)$.

$$
\mathrm{P}(\mathrm{r})=\mathrm{N} \times \mathrm{r} .
$$

In Eq. (1a), $\mathrm{N}$ is related to $\mathrm{r}$, so that we obtain:

$$
P(r)=r^{i-D} .
$$

By applying one-dimensional box-counting, we can derive a relationship similar to Eq. (4). Let $\mathrm{J}_{\mathrm{j}}(\mathrm{j}=1,2,3, \ldots, \mathrm{M})$ be the magnitude threshold, and the series of earthquake occurrence events be $X_{t}(t=1,2,3, \ldots, Q)$, where $Q$ is the total observed data number or the total time length. The earthquake events with magnitude equal to or larger than the threshold $\left(X_{t}>J_{j}\right)$ would form a point-process $\mathrm{T}_{\mathrm{i}}^{\left(\mathrm{Jj}^{\mathrm{j}}\right)}\left(\mathrm{i}=1,2,3, \ldots, \mathrm{Q}^{\left(\mathrm{j}_{\mathrm{j}}\right)}\right)$. For example, if the magnitude threshold $\mathrm{J}_{\mathrm{j}}$ $=6$, then $\mathrm{T}_{\mathrm{i}}^{(\mathrm{Jj})}$ would form a "Large-quake point-process" (Fig. 2). 
In the range of total time length $(\mathrm{Q})$, we apply several different smaller time length as time scales $\varepsilon_{\mathrm{K}}(\mathrm{K}=1,2,3, \ldots, \mathrm{L})$. For one-dimensional box-counting, the time scale $\left(\varepsilon_{\mathrm{K}}\right)$ can be seen as the "line length", which is similar to the concept of the cube size $(\varepsilon)$ and the ratio scale (r) in section 2.1. By each $\varepsilon_{\mathrm{K}}$, the range of the total time length would be divided into small time units $\left(\mathrm{Q} / \varepsilon_{\mathrm{K}}\right)$. Regardless to the actual events $\left[\mathrm{T}_{\mathrm{i}}^{(\mathrm{j})}\right]$ occurred in a certain time unit, the time unit would only be counted once if there is an event occurred in it. Thus, we can compute the cumulative frequency $\mathrm{N}^{(\mathrm{j})}\left(\varepsilon_{\mathrm{K}}\right)$ of time unit that contains at least one event. Finally, we establish the occurrence probability of point events in the form as follows:

$$
\mathrm{P}^{(\mathrm{Jj})}\left(\varepsilon_{\mathrm{K}}\right)=\mathrm{N}^{(\mathrm{Jj})}\left(\varepsilon_{\mathrm{K}}\right) /\left(\mathrm{Q} / \varepsilon_{\mathrm{K}}\right)
$$

By Eq. (4), we obtain:

$$
\mathrm{P}^{(\mathrm{Jj})}\left(\varepsilon_{\mathrm{K}}\right)=\left(\varepsilon_{\mathrm{K}}\right)^{\mathrm{l}-\mathrm{D}} \text {. }
$$

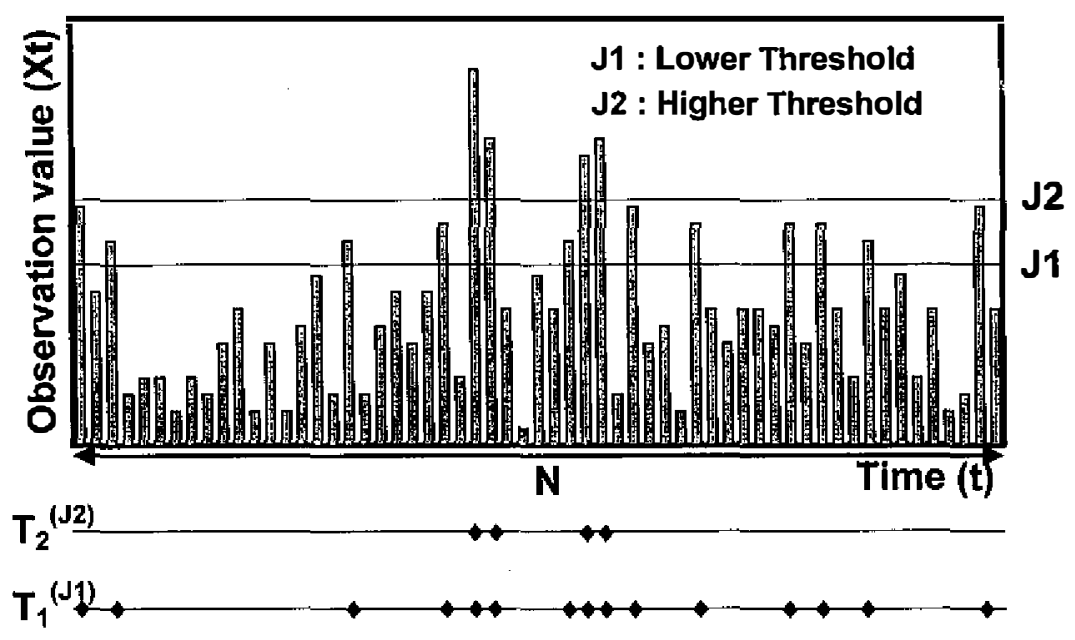

Fig. 2. Generation of point-process $\left[\mathrm{T}_{\mathrm{i}}^{(\mathrm{Jj} j}\right]$ having magnitudes equal to or larger than some threshold $\left(\mathrm{J}_{\mathrm{j}}\right)$. $\bullet$ expresses events with a given magnitude threshold. $\mathrm{T}_{1}^{\left(\mathrm{J}_{1}\right)}$ can be seemed as a smaller-quake point-process and $\mathrm{T}_{2}^{\left(\mathrm{J}_{2}\right)}$ as a larger-quake point-process. 


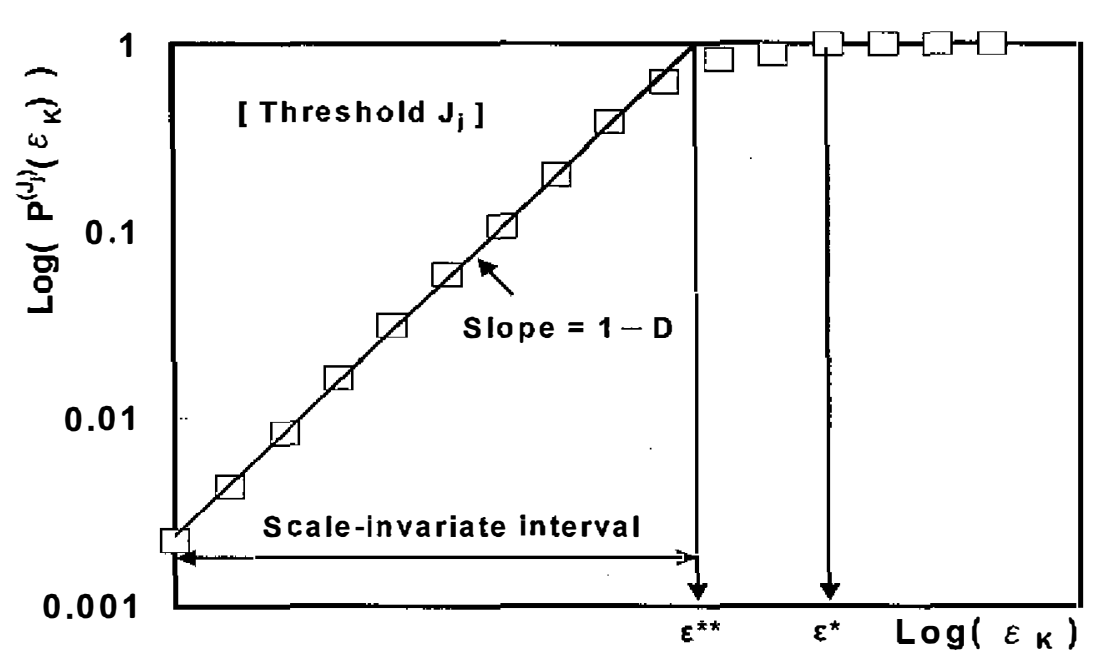

Fig. 3. Log-log plot of probability $\left[\mathrm{P}^{(j)}\left(\mathcal{E}_{\mathrm{K}}\right)\right]$ verse time scale $\left(\boldsymbol{\varepsilon}_{\mathrm{K}}\right)$ of earthquake time series under some magnitude threshold $\left(\mathrm{J}_{\mathrm{j}}\right)$. The slope of regression-line segment is (1-D), where $\mathrm{D}$ is the fractal dimension. The computed critical scale $\varepsilon^{*}$ is replaced by $\mathcal{E}^{* *}$ in this paper corresponding to an ideal time series with uniform fractal structure.

Combining Eq. (5a) and Eq. (5b), we get:

or

$$
\mathrm{N}^{(\mathrm{dj})}\left(\varepsilon_{\mathrm{K}}\right) /\left(\mathrm{Q} / \varepsilon_{\mathrm{K}}\right)=\left(\varepsilon_{\mathrm{K}}\right)^{1-\mathrm{D}}
$$

$$
\ln \left[\mathrm{N}^{(\mathrm{jj})}\left(\varepsilon_{\mathrm{K}}\right) /\left(\mathrm{Q} / \varepsilon_{\mathrm{K}}\right)\right]=(1-\mathrm{D}) \times \ln \left[\left(\varepsilon_{\mathrm{K}}\right)\right] .
$$

Figure 3 is a $\log$-log plot of a series of $\left[\mathrm{N}^{(\mathrm{Jj})}\left(\varepsilon_{\mathrm{K}}\right) /\left(\mathrm{Q} / \varepsilon_{\mathrm{K}}\right)\right]$ and $\left[\varepsilon_{\mathrm{K}}\right]$. In the plot, we constructed a regression straight-line segment in the scale-invariant interval, where slope is equal to (1-D). As the time scale increases and becomes equal to or larger than the critical scale $\mathcal{E}^{*}$, the probability of the critical scale $\mathcal{E}^{*}$ will reach 1 . It means earthquakes with magnitude equal to or larger than the threshold must occur as the time scale is equal to or larger than $\mathcal{E}^{*}$. In some practical cases, the straight line becomes curved when approaching probability 1 and is often interpreted as a combination of scale-invariant clustering and random events (Smalley et al. 1987). In order to define $\mathcal{E}^{*}$ more precisely, we extrapolate the regression-line with probability equal to 1 to replace $\mathcal{E}^{*}$ and define it as the critical scale, $\mathcal{E}^{* *}$. Therefore, the $\varepsilon^{* *}$ is an ideal critical time scale when fractal structure remained unchanged through the time axis. In this study, $\mathcal{E}^{* *}$ is a very important physical concept and can be applied as a reference for aseismatic evaluation. 


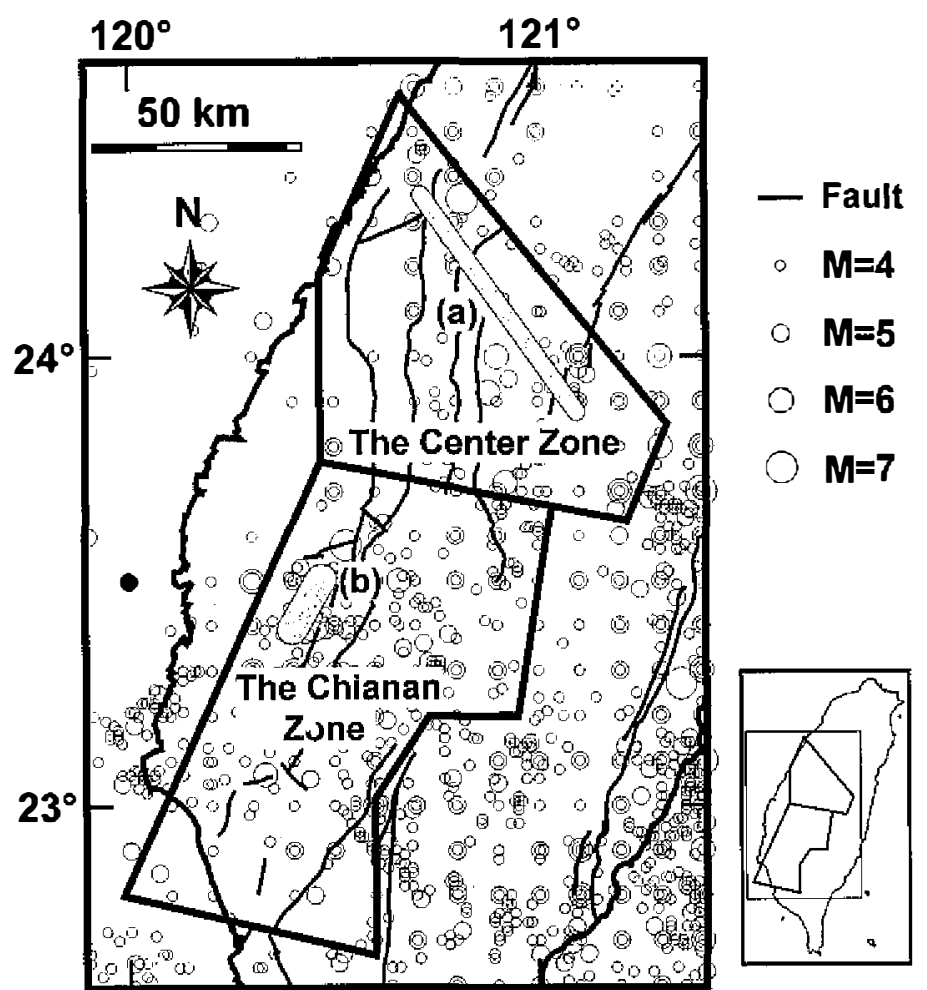

Fig. 4. Map showing the two seismogenic zones of Taiwan. The two gray areas, (a) Sanyi-Puli seismic belt and (b) Chianan seismic belt, are the belts concentrated in seismic activities.

\section{APPLICATION TO TWO SEISMOGENIC ZONES IN TAIWAN}

\subsection{Seismic Background}

Taiwan is situated in the boundary between the Euroasia plate and the Philippine Sea plate. It is an area with a high level of seismicity where global and local seismic networks and several previous instrumental observations (Hsu 1961) have provided a continuous record of events since 1900. Due to the difference between magnitudes recorded by TTSN (Taiwan Telemetered Seismographic Network; duration time magnitude, $M_{D}$ from 1973 to 1990) and CWBSN (Central Weather Bureau Seismic Network; local magnitude, $M_{L}$ since 1991), the earthquake catalog has been calibrated by applying the local magnitude $\left(\mathrm{M}_{\mathrm{L}}\right)$. According to the magnitude resolution of seismic networks, the earthquake catalog can be mainly divided into two periods: from 1900 to 1972 and from 1973 till today (Wang and Shin 1998). Catalog 
from 1900 to 1972 only contains large earthquakes with $\mathrm{M}_{\mathrm{L}} \geq 4$ and the locations are assigned at $10 \times 10 \mathrm{~km}$ grid positions due to the limited hypocenter determination ability. Catalog from 1973 till today contains earthquakes with $M_{L} \geq 2$ and the locations determined by the CWBSN can be constrained to an accuracy within 2-km inland and 5-km offshore by using widespread stations and modern instruments. This study is utilized earthquake data of $M_{L} \geq 4$ from 1900 to 1999.

We consider two seismogenic zones defined by Cheng (1997) based on regional tectonic framework, distribution of active faults, seismicity and earthquake characteristics. They contain intra-plate earthquakes shallower than $35-\mathrm{km}$ in depth and are renamed as the Center zone and the Chianan zone in this study (Fig. 4). The Center zone contains a Sanyi-Puli seismic

(a) Probability vs. Time Scale for the Center Zone

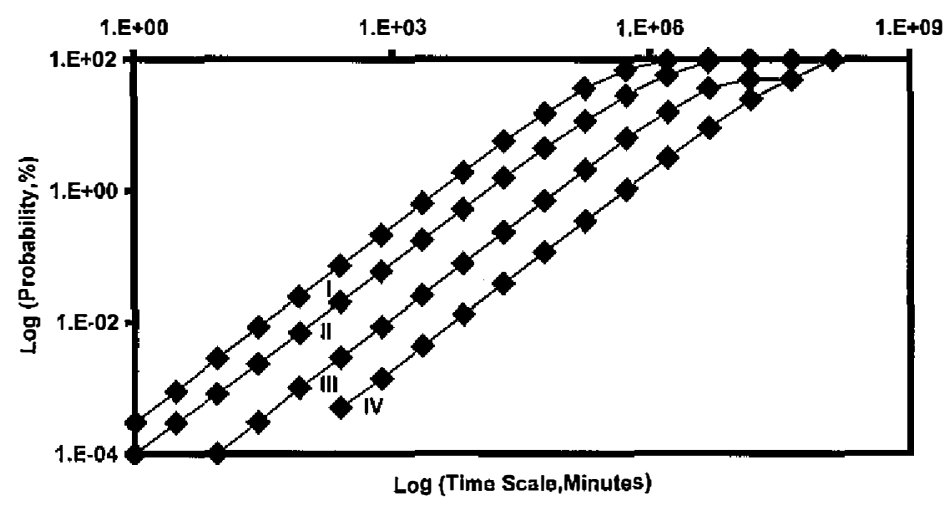

(b) Probabllity vs. Time Scale for the Chlanan Zone

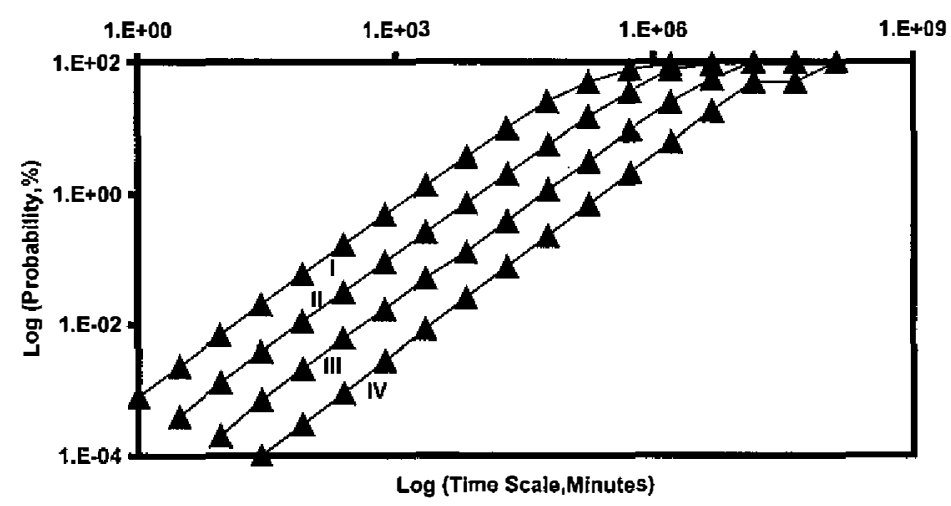

Fig. 5. Threshold fractal analysis of the Center and Chianan zones. Labels I, II, III and IV correspond to magnitude thresholds $\mathrm{J}_{\mathrm{j}}=4,5,6$ and 7 respectively. 
Table 1. Summarized results of threshold-fractal analysis of the Center and Chianan zones. $\mathrm{D}$ is fractal dimension; $\mathcal{E}^{* *}$ is the critical time scale considered here as the "lower limit" of recurrence period and $\mathrm{P}_{\mathrm{C}_{n}}$ is the value of intercept probability.

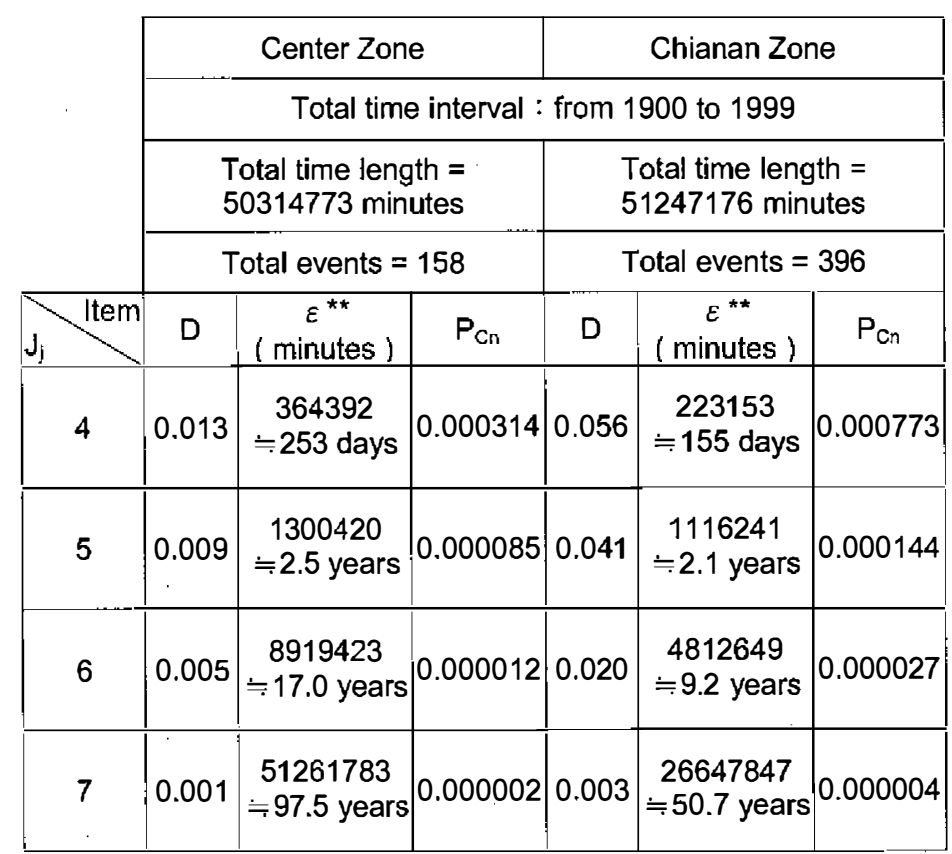

active zone. It's Gutenberg-Richter relationship is $\log N=2.553-1.087 \times \mathrm{M}$, and the magnitude ranges from $M_{L}=4$ to $M_{L}=7$. In this zone, the Chi-Chi earthquake of $M_{L}=7.3$ occurred in 21 September 1999 with the surface ruptures extended more than $105-\mathrm{km}$, the fault cliffs elevated several meters high, and the maximum PGA (Peak Ground Acceleration) greater than $1 \mathrm{G}$ (CGS 1999). The Chianan zone contains a Chianan seismic active zone. It's GutenbergRichter relationship is $\log N=3.107-1.117 \times \mathrm{M}$ and the selected earthquake ranges from $\mathrm{M}_{\mathrm{L}}$ $=4$ to $M_{L}=7$. These two zones are located at mountain front with high ground deformation according to GPS surveyed near Chiayi and Tainan (Yu 1997).

Based on time resolution of the earthquake catalog, the event number and the total time length of the data set, we chose 1 minute as the smallest time scale. The time scale is increased by a factor of 3 up to $3^{17}$ (129140163 minutes or 245.53 years) during the computing process.

\subsection{Computation and Discussion}

Within this catalog, there are 158 events selected in the Center zone and 396 events in the Chianan zone. Results of the threshold-fractal analysis of the earthquake catalog of the Center 
TAO, Vol. 14, No. 1, March 2003
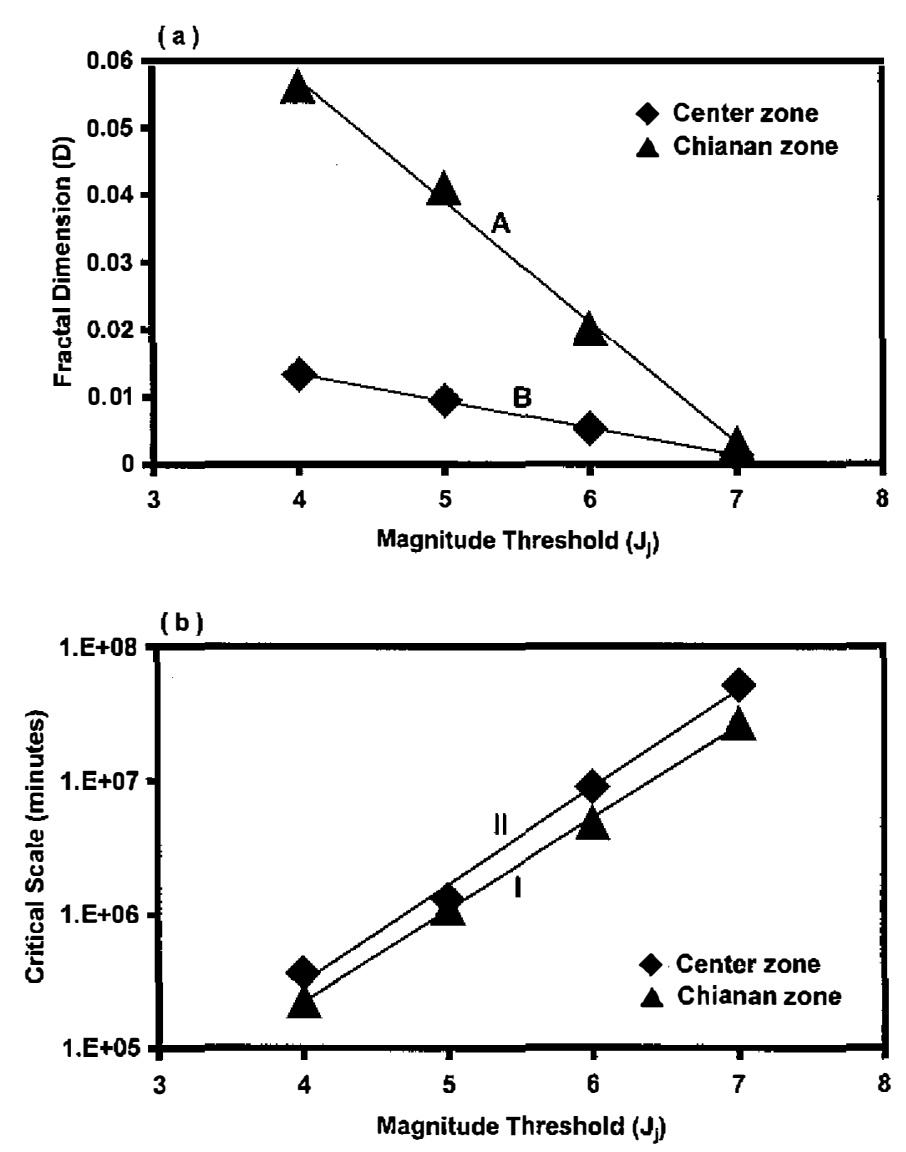

Fig. 6. Plots of (a) Fractal dimension (D) vs. magnitude threshold $\left(\mathrm{J}_{\mathrm{j}}\right)$ and (b) critical scale $\left(\varepsilon^{* *}\right)$ vs. magnitude threshold $\left(\mathrm{J}_{\mathrm{j}}\right)$. Fine lines are the best regression of the data. In (a), $D=-0.0179 \times\left(\mathrm{J}_{\mathbf{j}}\right)+0.1288$ for line $A$ and $\mathrm{D}=-0.004 \times\left(\mathrm{J}_{\mathrm{j}}\right)+0.0293$ for line B. In $(\mathrm{b}), \boldsymbol{\varepsilon}^{* *}=398.02 \times \mathrm{e}^{1.5809 \mathrm{j} \mathrm{j}}$ for line I and $\mathcal{E}^{* *}=379.7 \times \mathrm{e}^{1.6765 \mathrm{Jj}}$ for line II.

and Chianan zones are given in Figs. 5a, b respectively with data summarized in Table1.

The fractal dimension D decreases as the magnitude threshold $\left(\mathrm{J}_{\mathrm{j}}\right)$ increases (Fig. 6a). It implies that earthquake series is multifractal with respect to magnitude and the relationship is a negative linear in terms of the magnitude threshold. This result is different from the exponential decreasing of rainfall sequences (Lin 1998) and discloses some basic differences in the generation of these natural events. On the contrary, the critical scale $\mathcal{E}^{* *}$, increases with $\mathrm{J}_{\mathrm{j}}$ in an exponential manner (Fig. 6b).

Let the number of clusters ( $\mathrm{Cn}$ ) be the frequency computed by time scale of 1 minute. It is usually the same as the total number of events while time resolution is 1 minute. Then we can compute the probability $\left(\mathrm{P}_{\mathrm{Cn}_{\mathrm{n}}}\right)$ by $(\mathrm{Cn} / \mathrm{Q})$, where $\mathrm{Q}$ is the total time length of 1-minute resolution. 

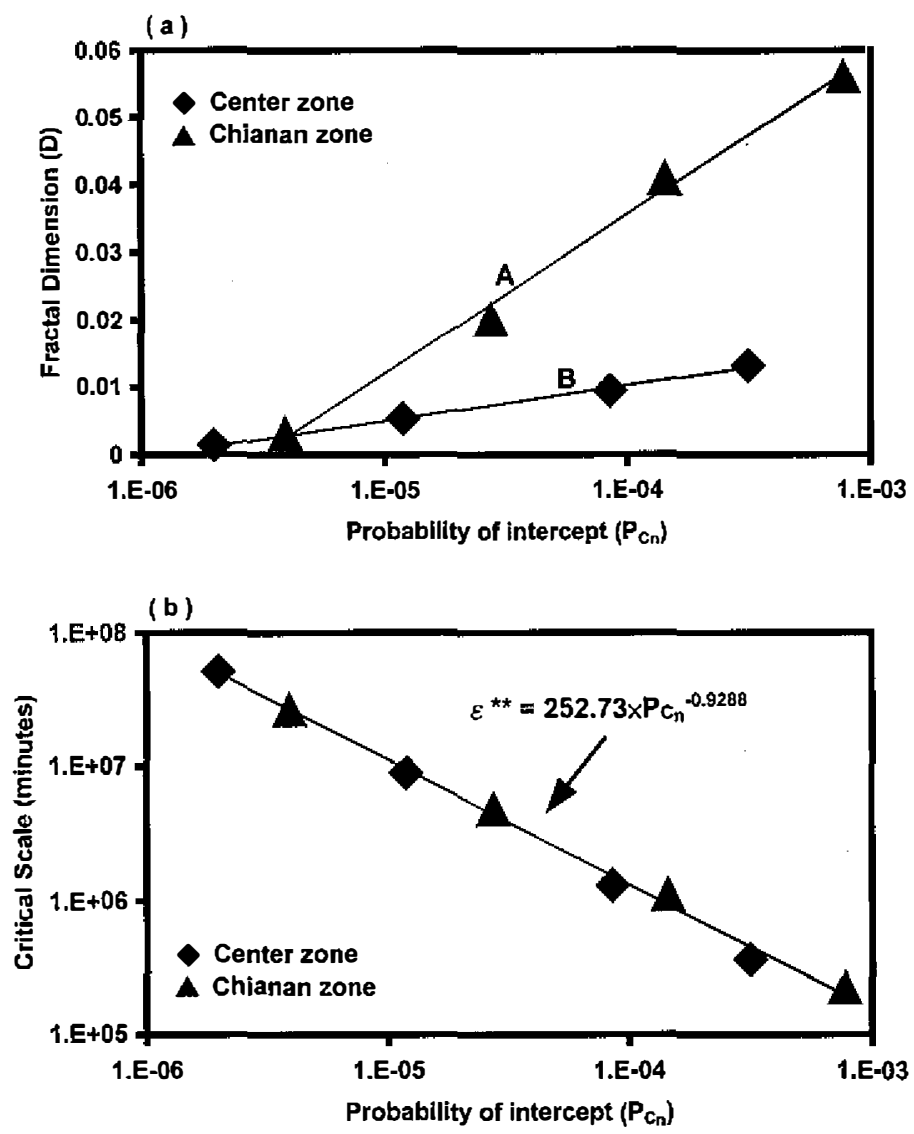

Fig. 7. Plots of (a) fractal dimension (D) vs. intercept probability $\left(\mathrm{P}_{\mathrm{Cn}_{n}}\right)$, and (b) critical scale ( $\left.\mathcal{E}^{* *}\right)$ vs. intercept probability $\left(\mathrm{P}_{\mathrm{Cn}}\right)$. In $(\mathrm{a})$, fine lines are the best regression lines with $\mathrm{D}=0.0102 \times \operatorname{Ln}\left(\mathrm{P}_{\mathrm{Cn}}\right)+0.1297$ for line $\mathrm{A}$ and $\mathrm{D}=0.0023 \times \operatorname{Ln}\left(\mathrm{P}_{\mathrm{C}_{\mathrm{n}}}\right)+0.0316$ for line $\mathrm{B}$. In (b), all data fall on one regression line.

As shown in Fig. 3, $P_{C n}$ equals to the intercept probability where $\log (1)=0$. Fig. 7a plots fractal dimension verse $\mathrm{P}_{\mathrm{Cn}}$ and the trends show different logarithmic increases with different time intervals and spatial zones. Smalley et al. (1987) indicated that the more isolated are the clusters, the smaller is the value of $\mathrm{D}$. In other words, if $\mathrm{Cn}$ is relatively smaller (or $\mathrm{P}_{\mathrm{Cn}_{\mathrm{n}}}$ is smaller) and more isolated in a certain time interval, it would form sparser clusters, and the recurrence periods of clusters would become longer with relative smaller fractal dimension. As $\mathbf{J}_{\mathbf{j}}$ becomes large, events will then occur sparsely, forming isolated clusters with large $\mathcal{E}^{* *}$ along the time axis with a smaller fractal dimension. 
TAO, Vol. 14, No. 1, March 2003

\subsection{Conclusion}

In this study, we found that when $\mathrm{J}_{j}=4$, the $\mathrm{D}$ value of the Chianan zone is much larger than that of the Center zone. D values of the two zones become almost equal when $\mathrm{Jj}_{j}=7$ (Fig. 6a). Thus, we may predict that there is no earthquake clustering in these two zones when magnitude $\mathrm{J}_{\mathrm{j}}$ is equal to or larger than 7 (e.g. the Chi-Chi earthquake). In Fig. $6 \mathrm{~b}$, all of the $\varepsilon^{* *}$ values in the Chianan zone are smaller than those in the Center zone and two regression lines are almost parallel. These results indicate that earthquake clustered more frequently and continuously in the Chianan zone than in the Center zone, especially for earthquakes with smaller magnitude. For larger earthquakes, the temporal behaviors of these two zones are similar. We may conclude that fault activities are more active in the Chianan zone than in the Center zone. The accumulated tectonic stress may be released through seismic activities more rapidly in the Chianan zone than in the Center zone.

Figure $7 \mathrm{~b}$ is the $\log -\log$ plot of $\varepsilon^{* *}$ versus $\mathrm{P}_{\mathrm{Cn}}$, and the entire data set is regressed by a power function:

$$
\varepsilon^{* *}=252.73 \times \mathrm{P}_{\mathrm{Cn}}^{-0.9288} .
$$

Substituted by Eq. (6), Eq. (7) becomes

$$
\varepsilon^{* *}=252.73 \times\left[\left(\varepsilon_{\mathrm{K}}\right)^{1-\mathrm{D}}\right]^{-0.9288} .
$$

Since $\varepsilon_{\mathrm{K}}=1$, the value of $\varepsilon^{* *}$ does not change with $\mathrm{D}$. This is the most significant result of this study in that the $\varepsilon^{* *}$ can be found if we only know how many events with magnitude equal to or larger than a critical $\mathrm{Jj}_{\mathrm{j}}$ had occurred, and the duration of total time length we want to include. Using these equations, we can easily extend the application into higher or lower magnitudes, such as $M_{L} \leq 1$ or $M_{L} \geq 8$, when historical earthquake records were not complete.

Finally, $\varepsilon^{* *}$ can be interpreted as the "lower limit" of seismic recurrence period when designing the aseismatic strength for buildings or civil constructions in their designed life. For an example, in the Chianan zone, earthquakes with $M_{L} \geq 7$ will occur when the time scale is equal to or larger than 50.7 years (Table 1). If we are to design a construction for more than 50 years in the Chianan zone, the aseismatic strength should be "at least" able to withstand vibrations caused by earthquakes with $M_{L}=7$.

Acknowledgements The authors thank Dr. T. Y. Lee for his support of the fractal analysis conception, and are greatly indebted to Mr. C. T. Cheng for providing original digital maps of seismogenic zones. They also appreciate the anonymous reviewers for critical reading. The research was supported by the National Science Council of Taiwan under the grant NSC 892119-M-017-001. 


\section{REFERENCES}

Aki, K., 1981: A probabilistic synthesis of precursory phenomena. In: Simpson, D.W. and Richards, P.G. (Eds), Earthquake Prediction. American Geophysical Union, Washington, D.C., pp. 566-574.

Cantor, G., 1883: Grundlagen einer allgemeinen Mannigfaltigkeitslehre. Mathematische Annalen, 21, 545-591.

CGS, 1999: Report of geological investigation of the 921 Chi-Chi earthquake. Central Geological Survey, Ministry of Economic Affairs, Taiwan R.O.C., 350pp.

Chen, Y., L. Chen, Z. J. Liu, and R. S. Wu, 1998: A new fractal approach to the clustering of earthquakes: Physical fractal. Bulletin of Seismological Society of America, 88 (1), 8994.

Cheng, C. T., 1997: Seismic hazard analysis in Taiwan region based on the new seismogenic zones. M.Sc. thesis, Department of Applied Geophysics, National Central University, Taiwan (in Chinese; 131 pp.).

Cheng, Y.C., P. J. Lee, and T. Y. Lee, 1999: Self-similarity dimensions of the Taiwan island landscape. Computers \& Geosciences, 25, 1043-1050.

Evernden, J. F., 1970: Study of regional seismicity and associated problems. Bulletin of Seismological Society of America, 60, 393-446.

Falconer, K., 1993: Fractal Geometry: Mathematical Foundations and Applications. John Wiley \& Sons, New York, $288 \mathrm{pp}$.

Gutenberg, B., and C. F. Richter, 1944: Frequency of earthquake in California. Bulletin of Seismological Society of America, 34, 185-188.

Hsu, M. T., 1961: Seismicity in Taiwan (Formosa). Bull. Earth . Res. Int., Tokyo Univ., 39, 831-847.

Isham, V., 1993: Statistical aspects of chaos: a review. In: Barndorff-Nielsen, O. E., Jensen, J. L., Kendall, W. S. (Eds), Networks and Chaos-Statistical and Probabilistic Aspects. Chapman \& Hall, London, pp. 134-136.

Kagan, Y. Y., and D. D. Jackson, 1991: Long-term earthquake clustering. Geophysical Journal International, 104, 117-133.

Lee, T. Y., 1995: Fractal structure of tempo-spatial geo-hydrologic variation and its applications. $\mathrm{Ph}$. D. dissertation, National Cheng Kung University, Tainan, Taiwan, pp. 17-33 (in Chinese).

Lin, S. C., 1998: The behavior of hydrologic variables in time-scale by Cantor set concept. Journal of Taiwan Water Conservancy, 46 (2), 85-96.

Mandelbrot, B. B., 1967: How long is the coast of Britain? Statistical self-similarity and fractional dimension. Science, 156, 636-638.

Mayer, L., 1992: Fractal characteristics of desert storm sequences and implications for geomorphic studies. Geomorphology, 5, 167-183.

Mazzarella, A., 1998: The time clustering of floodings in Venice and the Cantor dust method. Theoretical and Applied Climatology, 59 (3/4), 147-150.

Ogata, Y., 1988: Statistical models for earthquake occurrences and residual analysis for point 
processes. Journal of the American Statistical Association, 83 (401), 9-27.

Olsson, J., J. Niemczynowicz, R. Berndtsson, and M. Larson, 1992: An analysis of the rainfall time structure by box counting - some practical implications. Journal of Hydrology, 137, 261-277.

Papadopoulos, G. A., and V. Dedousis, 1992: Fractal approach of the temporal earthquake distribution in the Hellenic Arc - Trench System. Pure and Applied Geophysics, 139 (2), 269-276.

Peitgen, H. O., H. Jurgens, and D. Saupe, 1992: Chaos and Fractals: New Frontiers of Science. Springer-Verlag, New York, 984 pp.

Rasband, S. N., 1990: Chaotic Dynamics of Nonlinear Systems. John Wiley \& Sons, New York, $230 \mathrm{pp}$.

Smalley, R. F., J.-L. Chatelain, D. L. Turcotte, and R. Prevot, 1987: A fractal approach to the clustering of earthquakes: applications to the seismicity of the New Hebrides. Bulletin of Seismological Society of America, 77 (4), 1368-1381.

Stoyan, D., and H. Stoyan, 1994: Fractals, Random Shapes and Point Fields: Methods of Geometrical Statistics. John Wiley \& Sons, New York, 406 pp.

Tsai, H., 1999: The study on earthquake reocsurrence in Chiayi-Tainan area, Taiwan. Department of Geography, National Taiwan University, Journal of Geographical Science, 25, 111-126.

Turcotte, D. L., 1992: Fractal and Chaos in Geology and Geophysics. Cambridge University Press, Cambridge, $221 \mathrm{pp}$.

Yu, S. B., Y. S. Chen, and L. C. Kuo, 1997: Velocity field of GPS stations in Taiwan area. Tectonophysics, 274, 41-59.

Wang, J. H., 1991: A note on the correlation between b-value and fractal dimension from synthetic seismicity. Terrestrial, Atmospheric and Oceanic Sciences, 2, 317-329.

Wang, J. H., and C. W. Lee, 1995: Fractal characterization of an earthquake sequence. Physica , 221 (A), 152-158.

Wang, J. H., 1996: Multifractal measures of time series of Ms $\geq 7$ earthquakes in Taiwan. Journal of the Geological Society of China, 39 (1), 117-123.

Wang, C. Y., and T. C. Shin, 1998: Illustrating 100 years of Taiwan seismicity. Terrestrial, Atmospheric and Oceanic Sciences, 9, 589-614. 